\title{
Drag Analysis of Autonomous Vehicles in Different Arrays using CFD simulations
}

\author{
Filipe Fabian Buscariolo ${ }^{1}$, Julio Cesar Lelis Alves ${ }^{5}$, Flavio Koiti Maruyama ${ }^{3}$, Leonardo D. \\ Volpe $^{4}$, and Felipe C. Magazoni ${ }^{2}$ \\ ${ }^{1}$ Imperial College London / NDF-USP \\ ${ }^{2}$ Thermax / NETeF-USP \\ ${ }^{3} \mathrm{UFABC}$ \\ ${ }^{4} \mathrm{GM}$ North America \\ ${ }^{5}$ Technical Consultant \\ E-mails: f.fabian-buscariolo16@imperial.ac.uk, jclalves1@gmail.com, \\ flavio.koiti@gmail.com, leonardo.volpe@gm.com, felipe.magazoni@gmail.com
}

\begin{abstract}
Cargo trucks are one of the most important and flexible ways of moving cargo within inlands. In some countries, such as Brazil, the economy relies on them to transport all kinds of products, from field and factory to consumer. In order to reduce freight prices, beside route optimization, truck manufactures started to focus on the aerodynamics development of those vehicles, in order to improve the efficiency, reducing fuel consumption and emissions. Although the truck aerodynamics development is important, most vehicles are not manufactured or don't consider the truck trailer, which plays a key role in the full aerodynamics performance of the truck, once it might increase the front area and also change the overall aero performance.
\end{abstract}

Considering the fact that only few Wind Tunnel facilities in the world are capable of testing a full truck with trailer, the use of Computational Fluid Dynamics (CFD) simulations for the evaluation of the full truck system became the best solution in order to improve the aerodynamic performance of those vehicles. The study here presented proposes the CFD evaluation of a full truck, focusing on both truck and trailer design, evaluating different body styles and also evaluating the performance of exterior aerodynamic parts assembled on the vehicle.

Results are presented as a comparative between the baseline full truck and conceptual designs in terms of drag coefficient increments and also an image comparison between the baseline case and best case in terms of drag performance. This work wants to present an optimized geometry for the truck trailer, without losing cargo capacity, combined with the truck aerodynamics and extra improvements, pointing out that aerodynamics increments can't be added as a simple sum of factors.

\section{INTRODUCTION}

Automotive vehicle is one of the most important way of transporting people and cargo within inlands. They are divided mainly into two distinct groups passenger vehicles, such as cars and commercial vehicles such as road trucks. Estimations from the US publisher Ward's shows that the number of vehicles worldwide in 2016 was around 1.3 billion vehicles, 11 times more than 
the number in 1960. Among the total number in of vehicles in 2016, approximately $27 \%$ are commercial vehicles. Some countries concentrate the major part of the cargo and passengers transportation on road, such as Brazil with 58\% and Australia with 53\%. Considering Brazil, $75 \%$ of the country production is transported on road mainly by truck.

One of the major drawbacks of commercial vehicles are the combustion gases emissions higher than cars. Even considering green polices around the world regulating emissions and efficiency of vehicles such as EURO for European Marketing and the INOVAR Auto for the Brazilian market highlighted by BUSCARIOLO et. al. (2015), emissions of heavy-duty vehicles have grown by $2.4 \%$ a year since 2000, according to INTERNATIONAL ENERGY AGENCY (2018), faster than any other transport mode. At the same time, energy consumption has risen by $2.6 \%$ a year and trucks account for more than $90 \%$ of the growth in energy consumption. The main reason for this growth is the boost on road freight transport by trucks and vans due to the economy growth mainly in USA and China. The majority of those vehicles rely on petroleum products, elevating the share of diesel vehicles among the oil-based road fuels from $38 \%$ in 2000 to $44 \%$ in 2017.

Aiming to improve the trucks efficiency in terms of fuel economy and emission rates, commercial vehicle manufactures started to perform aerodynamic studies in order to optimize the drag coefficient and frontal area values. For wind tunnel tests of the full truck, considering the trailer, only a few facilities in the world, such as the DNW Wind Tunnel in Netherlands and the National Full-Scale Aerodynamics Complex (NFAC) Ames Research Centre in the USA as stated by PATTEN et. al. (2012). An alternative solution is the use of CFD simulations in order to evaluate the aerodynamic performance of the full truck, as the numerical domain can be discretized with sufficient large dimensions that avoid blockage effects. Computational simulations can be performed at early stages of the project avoiding costly changes at advanced phases and also offers a reliable tool for evaluating proposed exterior parts and geometry changes without the need of prototype manufacture. Combined with wind tunnel test, CFD provide a guideline of the critical areas in terms of aerodynamic sensitivity, improving the efficiency of the test by leading the engineers work on more effective aerodynamic solutions.

The study of HYVÄRINEN (2015) presents a virtual evaluation of the blockage effects when performing a test in a closed wind tunnel against open road conditions with a truck equipped with trailer in three different conditions and results indicate different force distribution, flow features and drag coefficient results when comparing the two conditions. The main conclusion we can draw is that even wind tunnel tests might mitigate flow effects if blockage conditions are not respected.

A summary of the techniques used to evaluate and develop the aerodynamic properties of trucks equipped with trailer is presented in the report by PATTEN et. al. (2012). Experimental facilities are presented and discussed in terms of benefits and points to improve as well as CFD simulations and other methodologies. The study presents good references in terms of wind tunnels however the CFD methods advanced with the years and provide much better results in more reliable time than what was presented in 2012.

Most experimental academic studies are performed with scaled model due to size limitation and one interesting example is the work performed by SHUKRI and AKRAM (2013) where they performed an experimental study on a 1/30 scale model of a truck in order to evaluate the behaviour of adding flaps on the rear part of the trailer showing some drag improvements 
around $20 \%$, depending on both flap and vehicle yaw condition. CFD results performed by the author also show good correlation with experiments.

The compilation of studies presented by BUSCARIOLO et. al. (2016), where general aerodynamic enablers are presented with expected drag reduction performance aimed main for cars, we found an interesting solution applied to truck trailers which consists in an active flow control system by using plasma actuators leading to a potential drag reduction of $12 \%$. The use of aeroparts is related to the second study here presents and both phases are detailed as follows.

\section{OBJECTIVES}

The study first proposes a comparative drag coefficient analysis between four different truck body styles combined with the same trailer, in order to evaluate how the design evolution impacted on the aerodynamic performance of the full vehicle. All truck geometries simulated are open sources available at GrabCAD and are presented on Figure 1.
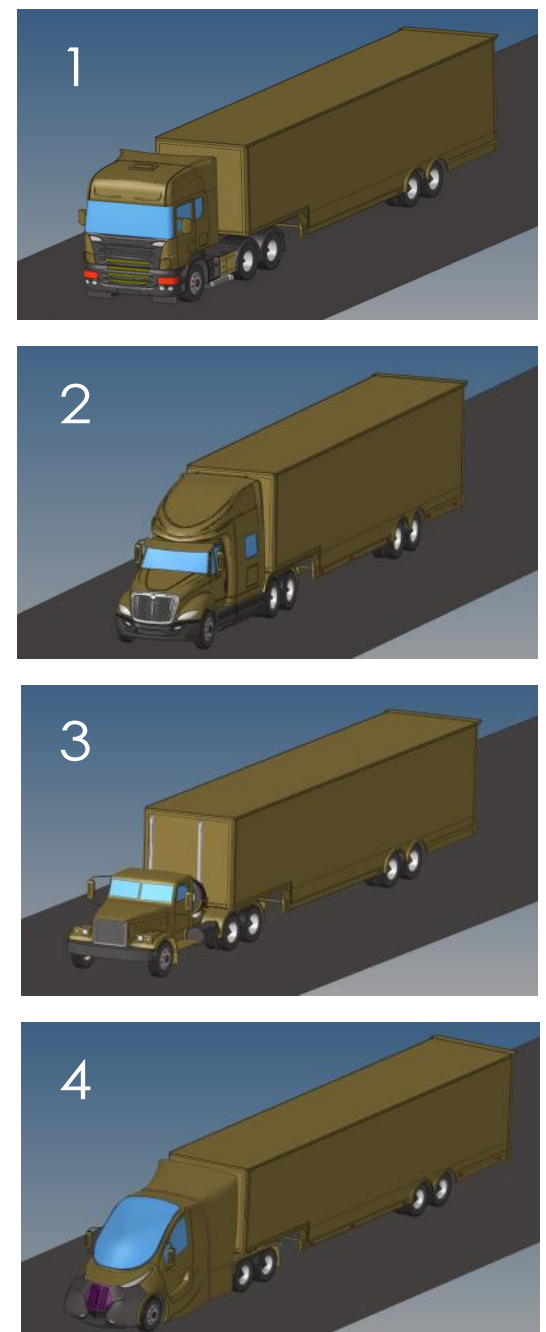

Figure 1. Open source geometries of the trucks evaluated in this study. From top to bottom: truck tractor with short face (1); truck tractor with extended face (2); truck tractor with extended face old design (3); truck tractor with extended face conceptual design (4). Available at GrabCad. 
The second portion of the study take into account the truck tractor body style with short face, very common cabin type for long haul and road trucks in Europe and South America. Baseline geometry and case considering additional parts proposed are presented on Figure 2.
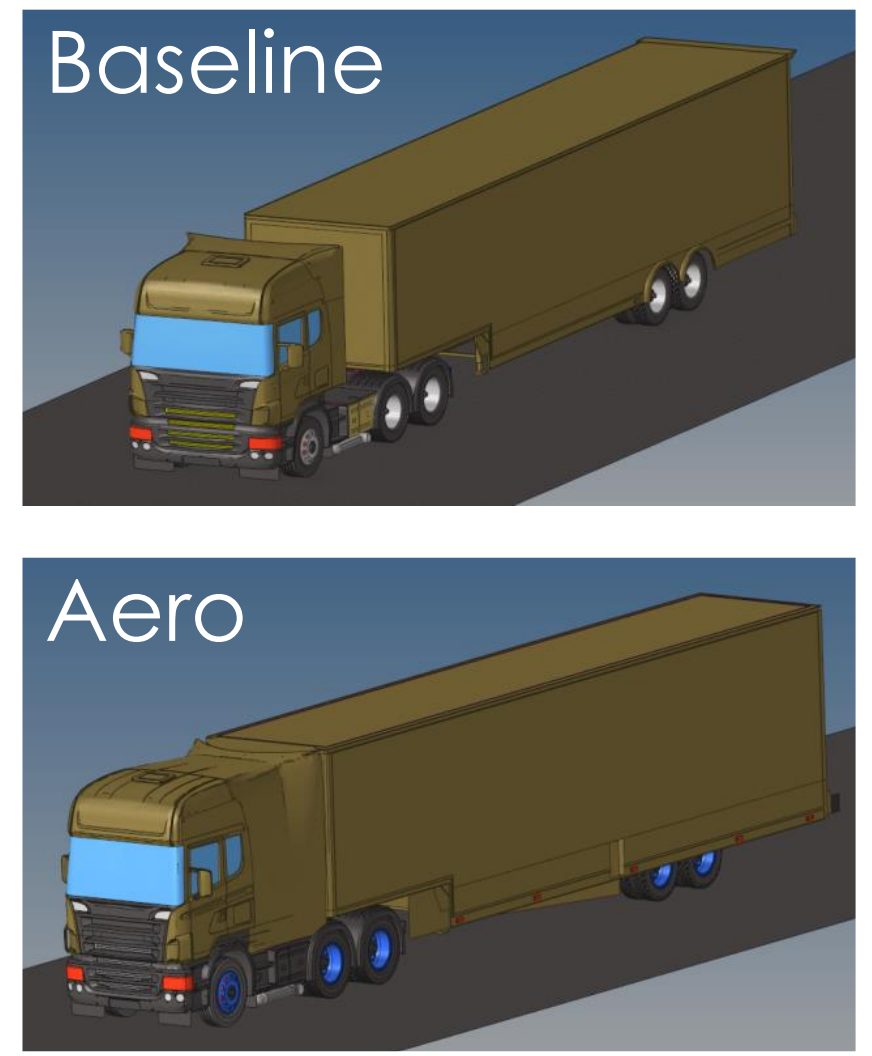

Figure 2. Open source geometries of the truck tractor with short face (Baseline) and truck tractor with short face with aerodynamic enablers (Aero) Available at GrabCad.

The proposed changes are: a flexible connection with spoiler between the truck and the trailer, deflectors for the truck wheel wake, air guides for the underbody trailer flow and trailer wheel cover, as presented on Figure 3.

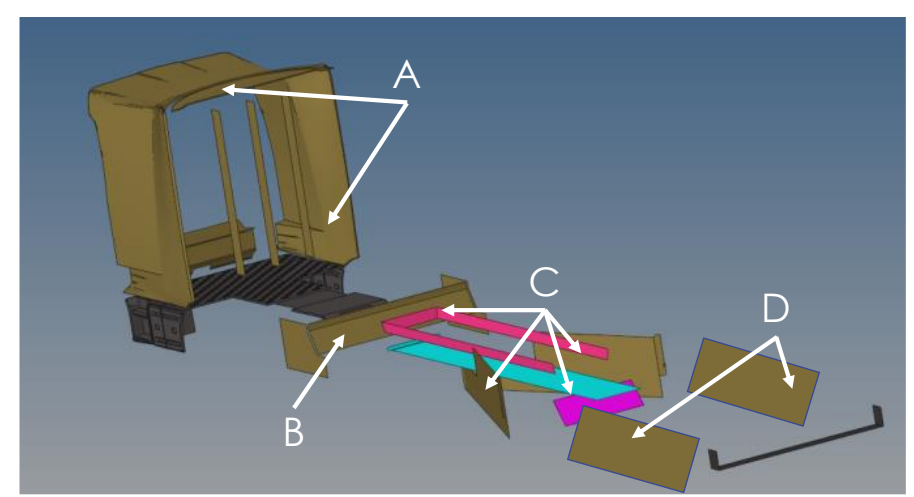

Figure 3. Conceptual enablers proposal for the Aero version of the truck tractor with short face: flexible connection with spoiler between the truck and the trailer (A); deflectors for the truck wheel wake (B); air guides for the underbody trailer flow (C); trailer wheel cover (D). 
Results are obtained by numerical simulations and values for the drag coefficient for all cases are presented in term of percentage, considering the truck tractor body style with short face as the baseline case.

\section{TECHNICAL FORMULATION}

We use the same Reynolds Averaged Navier-Stokes methodology detailed on the work of BUSCARIOLO et. al (2016) for automotive applications. Replacing the scalar variables on the fluid conservation law equation, together with the appropriate adaptations, we obtain the Navier-Stokes equations, which describe the motion of a fluid and its properties, and considering incompressible, isothermal Newtonian flow (density and viscosity are constants),

$$
\begin{gathered}
\nabla \cdot V=0 \\
\rho \frac{D V}{D t}=-\nabla P+\rho g+\mu \nabla^{2} V
\end{gathered}
$$

where $\mathrm{P}$ is pressure, $\mu$ is the viscosity, $\mathrm{V}$ is the velocity field and $g$ is the acceleration vector.

As the averaged Navier-Stokes Equations, basis of most of the computational CFD codes, compose an open equation system due to the additional Reynold's stress terms. Hence, a turbulence model needs to be incorporated in order to close the equation system. Turbulence models considered in this study are the $k-\varepsilon$ and k- $\omega$ SST models.

The $k-\varepsilon$ turbulence model is composed by two equations that come directly from the differential transport equations, in which $k$ represents the turbulent kinetic energy and $\varepsilon$ represents the turbulence dissipation rate. The two equations are written below [4] [9],

$$
\begin{gathered}
\frac{\partial \rho k}{\partial t}+\operatorname{div}\left(\rho k u_{r}\right)= \\
=\operatorname{div}\left(\frac{\mu_{t}}{\sigma_{k}} \operatorname{grad} k\right)+2 \mu_{t} E_{i j} E_{i j}-\rho \varepsilon \\
\frac{\partial \rho \varepsilon}{\partial t}+\operatorname{div}\left(\rho \varepsilon u_{r}\right)=\operatorname{div}\left(\frac{\mu_{t}}{\sigma_{\varepsilon}} \operatorname{grad} \varepsilon\right)+ \\
+C_{1 z} \frac{\varepsilon}{k} 2 \mu_{t} E_{i j} E_{i j}-C_{2 z} \rho \frac{\varepsilon^{2}}{k}
\end{gathered}
$$

where $\mu_{t}$ is the turbulent viscosity, $C_{1 z}, C_{2 z}, \sigma_{\varepsilon}$ and $\sigma_{k}$ are turbulence model constants.

Once the fluid behavior and properties are known, the next step is to calculate drag coefficient, which comes from the pressure acting over the vehicle due to the contact with air. Once the drag force (D) is an output from the Navier-Stokes solution, the drag coefficient of a vehicle, which is characterized by its frontal area $A$ and vehicle speed $V$ is expressed by the following equation below,

$$
c_{D}=\frac{2 D}{\rho V^{2} A}
$$




\section{METHODOLOGY}

In order to perform this study on CFD, a virtual wind tunnel is considered, with test section similar dimensions of $9.2 \mathrm{~m}$ for the width and $7 \mathrm{~m}$ for the height, with cross section area of $64.40 \mathrm{~m}^{2}$. In order to fully fit the trucks, the length considered for the test section is $48 \mathrm{~m}$.

The velocity imposed on the inlet and considered for the test is, a real road condition for a truck moving on a highway. Moving ground simulation was also considered for the tire-floor interface and atmospheric pressure boundary condition applied on the outlet. For the wind tunnel wall, symmetry boundary condition was applied and the turbulence intensity on the test section was imposed to be around $0.6 \%$, similar to well-controlled wind tunnels.

Table 1. Parameters for the simulations

\begin{tabular}{|l|c|}
\hline Wind tunnel & Virtually designed \\
\hline Air speed & $80 \mathrm{kph}$ \\
\hline Outlet pressure & Atmospheric pressure \\
\hline Turbulence intensity & $0.60 \%$ \\
\hline Boundary layer suction system & At the beginning of the test section \\
\hline Test Section dimensions & $(9.2 \times 7.0 \times 48.0) \mathrm{m}$ \\
\hline
\end{tabular}

Trailer dimensions are set to match a real operation vehicle, in accordance to the traffic regulation and is $13 \mathrm{~m}$ long, $3 \mathrm{~m}$ high and the width is $2.3 \mathrm{~m}$. For each of the truck evaluated, we have a different frontal area $\mathrm{A}$ and values are summarized on table 2.

Table 2. Frontal are for different truck body styles evaluated

\begin{tabular}{|l|c|}
\hline Truck Body Style & $\begin{array}{c}\text { Frontal } \\
\text { Area }\left(\mathbf{m}^{2}\right)\end{array}$ \\
\hline truck tractor with short face & 8.28 \\
\hline truck tractor with extended face & 7.71 \\
\hline truck tractor with extended face old design & 7.88 \\
\hline truck tractor with extended face conceptual design & 8.28 \\
\hline
\end{tabular}

For each case, surface mesh was discretized with average truck surface size of $10 \mathrm{~mm}$ with some refinements for critical areas such as mirrors, columns and grilles. For the volumetric mesh, the average number of cells reaches around 30 million, considering refinement boxes for the truck and wake and prism boundary layer for most of exterior components. 


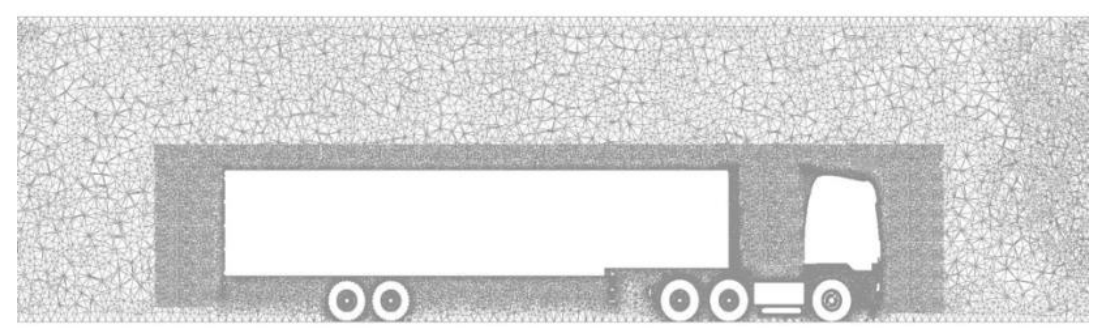

$+1$

Figure 4. Mesh details on the plane $\mathrm{Y}=0 \mathrm{~mm}$ for the short face truck.

Simulations are performed using the software Star-CCM+ on the Imperial College Cluster for sufficient time for the solution to reach physical convergence on a steady-state case.

\section{RESULTS}

Considering the 4 load cases evaluated for the different body styles and selecting the short face vehicle as the baseline, we found out that the best design is the extended face conceptual design, followed by the extended face. Results are presented as a percentage from the baseline vehicle.

Table 3. Summary of Drag Coefficient increments for all load cases evaluated

\begin{tabular}{|l|c|}
\hline Truck Body Style & $\begin{array}{c}\text { Delta Cd } \\
(\mathbf{\%})\end{array}$ \\
\hline BASELINE - truck tractor with short face & 1 \\
\hline truck tractor with extended face & $-19 \%$ \\
\hline truck tractor with extended face old design & $+41 \%$ \\
\hline truck tractor with extended face conceptual design & $-22 \%$ \\
\hline
\end{tabular}

Considering the results in Table 3, it's possible to conclude that the evolution of the design improved the aerodynamics and efficiency of the current trucks. We can also conclude that the extended face style provides a smoother flow transition and the trend shows aero performance improvement of at least $19 \%$.

Now evaluating the improvements in terms of the short face baseline with the proposed aero version, we obtained an improvement of $5 \%$ on the total drag, presented on table 4.

Table 4. Summary of Drag Coefficient increments for all load cases evaluated

\begin{tabular}{|l|c|}
\hline Truck Body Style & $\begin{array}{c}\text { Delta Cd } \\
(\boldsymbol{\%})\end{array}$ \\
\hline BASELINE - truck tractor with short face & 1 \\
\hline AERO & $-5 \%$ \\
\hline
\end{tabular}

Comparing the flow profile over the different designs presented on Figure 5, it is possible to identify an intense recirculation zone behind the cabin of the short face vehicle (1), mainly generated by the gap between the cabin and the trailer, leading to a negative impact on drag. 

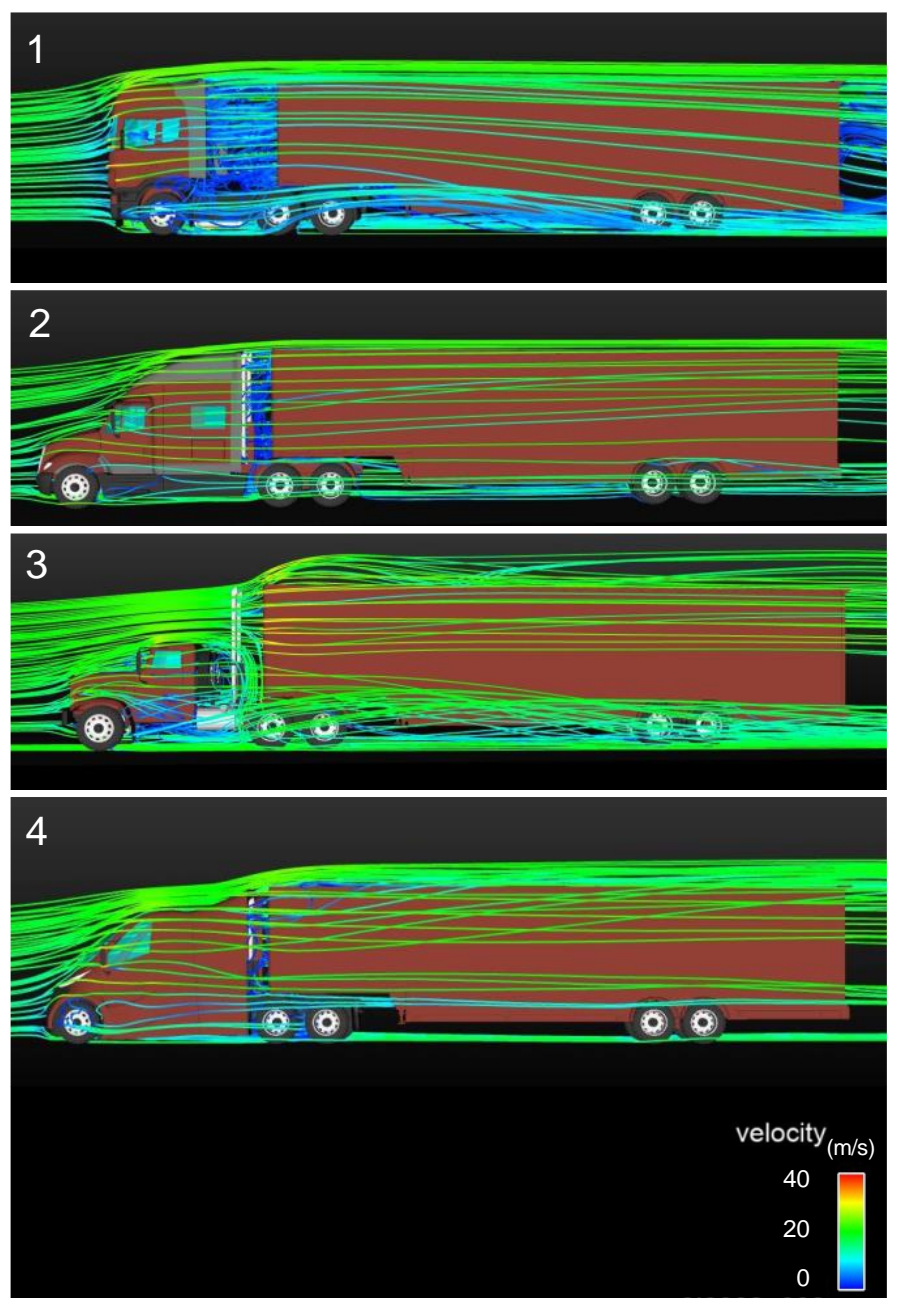

Figure 5. Streamline velocity contours over the truck surfaces evaluated: truck tractor with short face (1); truck tractor with extended face (2); truck tractor with extended face old design (3); truck tractor with extended face conceptual design (4).

We also observe that the trucks with better aerodynamic performance, ( 2 and 4$)$ have smother flow transition and the recirculation zone behind the cabin is smaller compared to the baseline case. The old design truck (3) presents a well-defined vortex on the back of the cabin, mainly generated by the absence of deflectors and the clear distance to the trailer. Those geometry characteristics lead to a fixed separation point at the end of the cabin with low-pressure gradient, increasing the intensity of the vortex and compromising the aerodynamic performance. The flow reaching the trailer on the truck (3) also has a separation bubble from the beginning to one quarter of the length of the trailer, which is not noticed on the other trucks mainly due to the design of the cabin that prevents this separation to happen. 

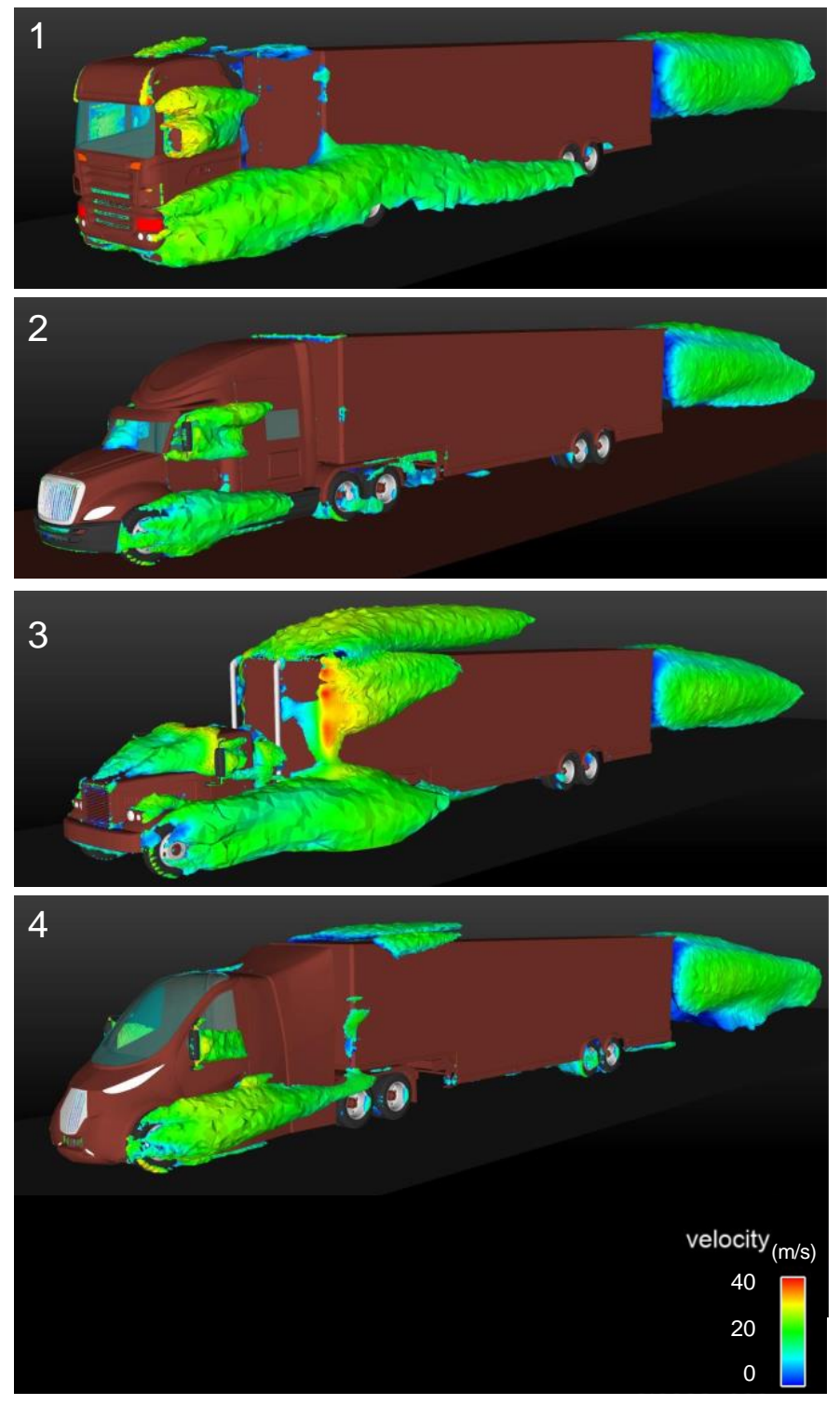

Figure 6. Wake profile colored by velocity on the truck surfaces evaluated: truck tractor with short face (1); truck tractor with extended face (2); truck tractor with extended face old design (3); truck tractor with extended face conceptual design (4).

Comparing the wake profiles, extracted for each body style and colored by velocity, we notice that the surface with better aerodynamic performance which are design (2) and (4) also have smaller turbulent wake regions, compared with baseline case. Although (4) has a bigger wake on the wheel and truck-trailer transition, there is a substantial reduction on the side mirror wake. We confirm flow separation prediction on the truck-trailer transition on old truck design (3), not only on top, but also on the lateral transition which, combined with a long wheel wake, contribute to increase the drag. The finds presented lead us to conclude that smaller wake size improve drag coefficient performance. 

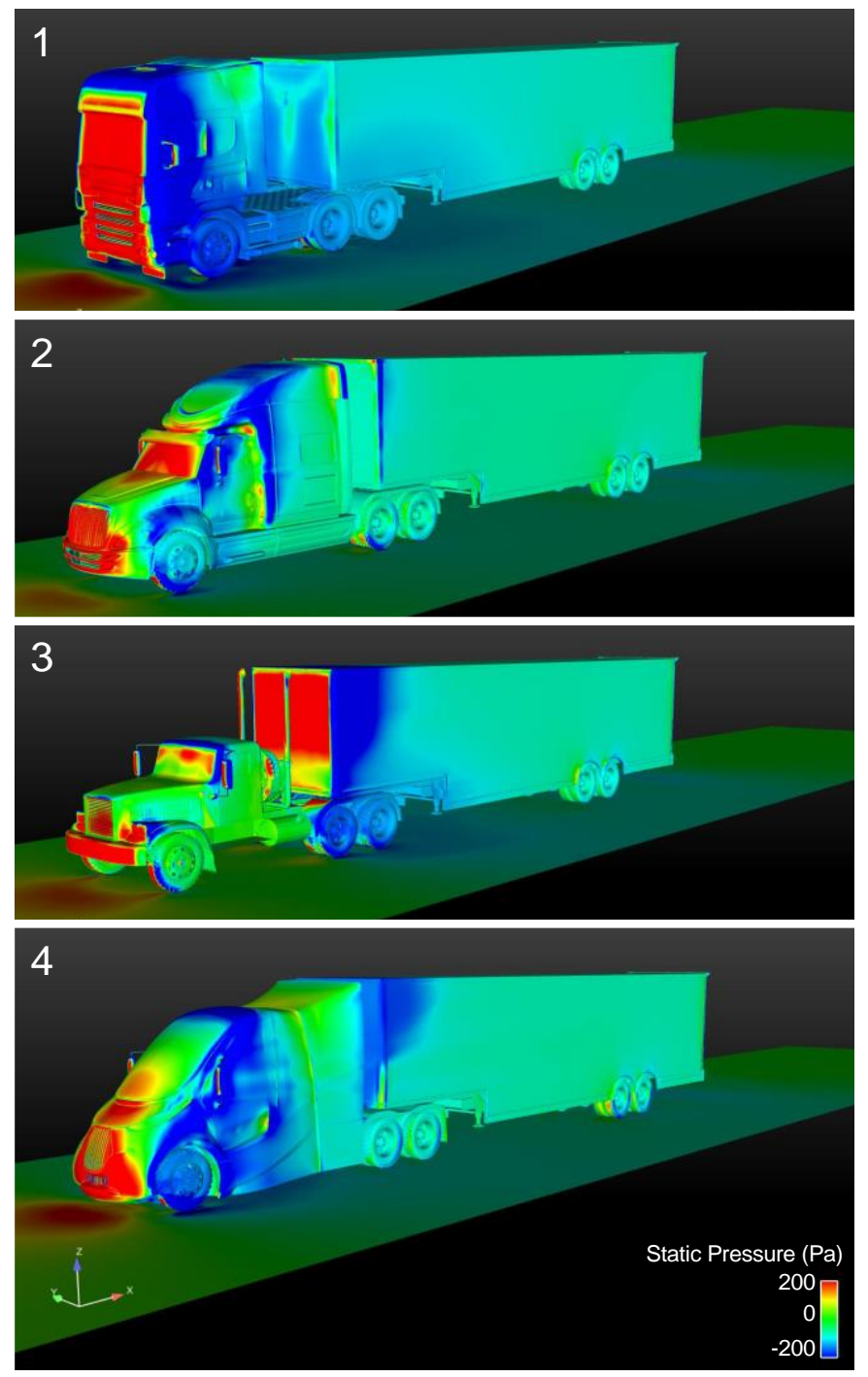

Figure 7. Static pressure contour on the truck surfaces evaluated: truck tractor with short face (1); truck tractor with extended face (2); truck tractor with extended face old design (3); truck tractor with extended face conceptual design (4).

Contour of static pressure is presented on Figure 7, where trucks considering extended face have a smoother flow transition, when compared to the short face (1) where most of the frontal area acts as a stagnation point. Surface and aerodynamic features on the frontal portion compensate of the short face (1) recover some drag performance for the far field flow. The old face design (3) has a strong stagnation on the trailer without any aerodynamic device to optimize the flow transition, decreasing its aerodynamic performance. The conceptual design (4) has an integrated windshield design which helps on reducing the pressure stagnation area, especially compared to extended face (2), where the windshield position creates another stagnation zone.

When analysing the improvements on the short face truck, used as benchmarking for the aerodynamic proposal presented, it's possible to conclude that the turbulent wake due to the recirculation zone behind the cabin is almost eliminated. This is the main change on the flow field over the vehicle within the aerodynamic parts study presented on Figure 8. 

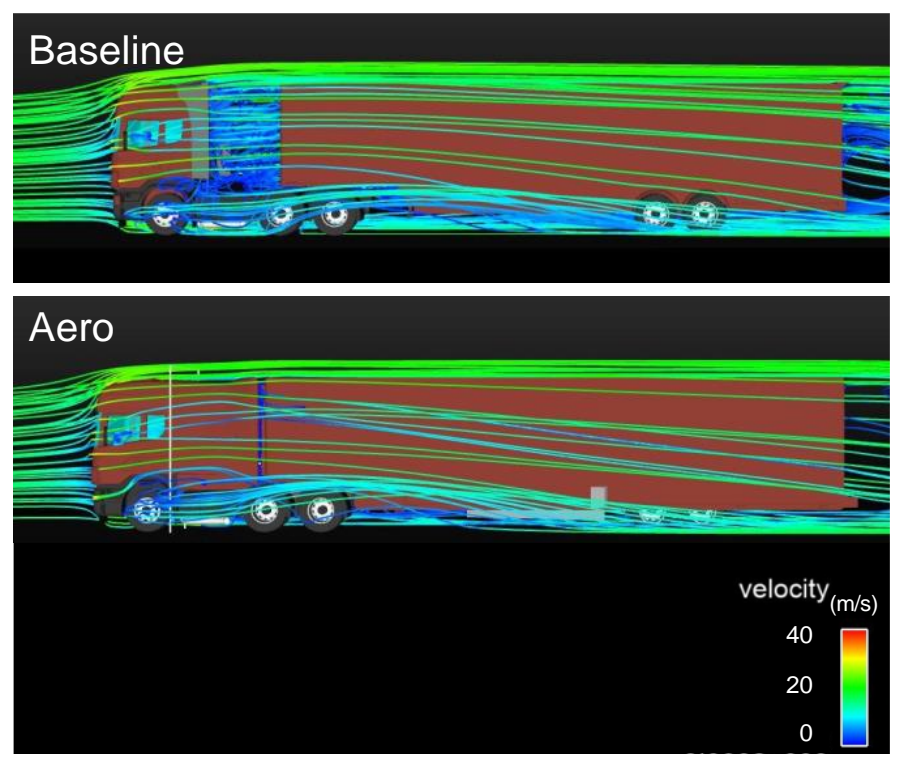

Figure 8. Streamline velocity contours over the truck tractor with short face (Baseline) and truck tractor with short face with aerodynamic enablers (Aero)

Analyzing the wake profile over the Baseline and Aero trucks on Figure 9, it possible to notice the reduction of the turbulent wake on the wheel region, mainly due to the underbody deflectors, improving the overall aerodynamic performance. There is also a slight reduction on the size of the mirror wake once the gap between the truck and trailer are now closed. For both cases, the wake behind the trailer looks similar, without any visible change in size or intensity.
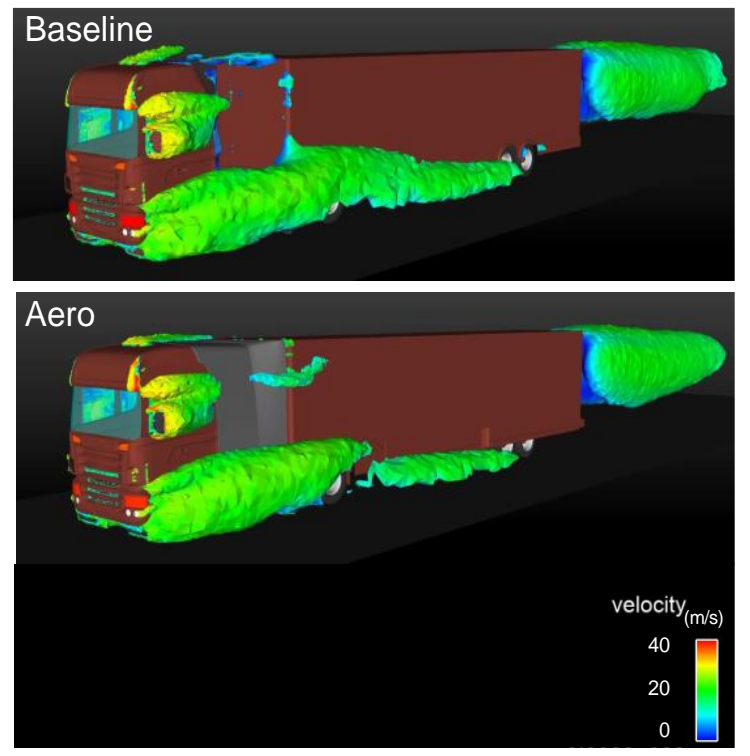

Figure 9. Wake profile colored by velocity on the truck tractor with short face (Baseline) and truck tractor with short face with aerodynamic enablers (Aero)

\section{CONCLUSION}

Within the emission and fuel economy requirements constantly increasing, aerodynamics is becoming a popular field of study in the commercial and cargo vehicles industries, aiming to 
reach the desired project performance. Among the most important commercial vehicles are the trucks, responsible for moving cargo inlands. Optimizing trucks performance also direct impact on the profit of companies and drivers.

Due to the size constrains few Wind Tunnel facilities in the world are able of testing trucks, so CFD simulations provide a suitable alternative aerodynamic evaluation. This study proposes the evaluation of a full truck, focusing on both truck and trailer design, evaluating four different body styles and also evaluating the performance of exterior aerodynamic parts assembled on the vehicle using computational simulations. The short face truck is considered as the baseline model for reference.

Results comparing different surfaces and body styles indicate that the extended face (2) and extended face conceptual design (4) body styles has a drag coefficient performance around $20 \%$ better than the short face, mainly due to smoother flow transition from the vehicle's surface to the trailer. The old design truck, although also presenting initial smoother transition has aero performance $41 \%$ worse than the baseline, justified by the fact of a strong vortex behind the cabin and separation bubble over the trailer.

When comparing the conceptual aero enablers on the baseline model, the main find is the reduction of the recirculation zone to a minimal size, leading to a drag improvement of 5\%.

We also conclude that the turbulent wake is an indicator of drag performance, where vehicles presenting bigger wakes usually have inferior aerodynamic performance, once those features are connected to chaotic flow behavior.

One of the main points we want to emphasize is that aerodynamics increments for different aerodynamic parts and surface changes can't be added as a simple sum of factors, once the flow physics is affected by the whole continuum.

\section{REFERENCES}

1. Buscariolo, F.F.;Magazoni, F., Wolf, M.,Maruyama F., Alves, J. C. L., Volpe, L. D., "Analysis of Turbulence Models Applied to CFD Drag Simulations of a Small Hatchback Vehicle", SAE Paper, No 2016-36-0201, Society of Automotive Engineers, 2016.

2. Buscariolo, F.F., Magazoni, F., Maruyama, F.K., Alves, J.C.L., Della Volpe, L.J. and Holl, A.F., "Aerodynamic Enablers Review for Automotive Applications", SAE Paper, No 201636-0203, Society of Automotive Engineers, 2016.

3. FLUENT 15, “User's Guide”, Fluent Inc., 2013.

4. Maruyama, F., Alves, J. C. L., Volpe, L. D., Magazoni, F., Buscariolo, F. F., "Wheel Design Sensitive Analysis on Drag of Small Sedan Vehicle", SAE Paper, No 2015-36-0168, Society of Automotive Engineers, 2015.

5. Tennekes, H.; Lumley, J. L.,"A First Course in Turbulence”, MIT Press, Cambridge, MA, 1972.

6. GrabCAD.com, https://grabcad.com/ 
7. Patten J, McAuliffe B, Mayda W, Tanguay B. Review of aerodynamic drag reduction devices for heavy trucks and buses. National Research Council Canada NRC Technical Report CSTT-HVC-TR. 2012 May 11;205:3.

8. Hyvärinen A. Investigation of blockage correction methods forfull-scale wind tunnel testing of trucks. Master Thesis, KTH, 2015

9. Shukri IA, Akram AM. Improvement of aerodynamics characteristic of heavy trucks. In3rd International Conference on Trends in Mechanical and Industrial Engineering (ICTMIE'2013) January 2013 Jan (pp. 8-9)

10. International Energy Agency accessed on May, 2019 https://www.iea.org/tcep/transport/trucks/ 